\title{
The Effect of Hemodynamic Parameters on Cerebral Oxygenization During Carotid Endarterectomy
}

\author{
Cihan Yücel ${ }^{1}$, MD; Serkan Ketenciler ${ }^{1}$, MD; Mete Gürsoy ${ }^{1}$, MD; Seray Türkmen², MD; Nihan Kayalar ${ }^{1}$, MD
}

DOI: $10.21470 / 1678-9741-2020-0398$

\begin{abstract}
Objective/Introduction: Near-infrared spectroscopy (NIRS) is a noninvasive technique to detect cerebral ischemia by monitoring changes in regional cerebral oxygenation $\left(\mathrm{rSO}_{2}\right)$ in the frontal lobes. However, there are no studies showing the changes in NIRS values in response to hemodynamic variations during stages of carotid endarterectomy (CEA) procedure and clinical implications of these changes. The aim of this study was to determine if hemodynamic changes affect NIRS values during carotid endarterectomy and if our results may help to provide strategies for hemodynamic management in these patients.

Methods: $A$ total of 50 consecutive patients undergoing CEA were prospectively included in the study. NIRS was measured at first minute after clamping of carotid artery, and then systolic blood pressure was increased above $150 \mathrm{mmHg}$. NIRS values from both hemispheres were recorded simultaneously at certain time points and were analyzed to evaluate the changes at different stages of operation and to assess
\end{abstract}

correlations with hemodynamic parameters.

Results: NIRS values on the right and left sides were correlated with systolic (right $P<0.001, R 2: 0.24$; left $P=0.02, R 2: 0.10$ ) diastolic (right $P<0.001, R 2: 0.36$; left $P=0.001, R 2: 0.18$ ) and mean (right $P<0.001$, R2:0.33; left $P=0.003, R 2: 0.17$ ) blood pressures when the patient was under general anaesthesia. NIRS values were significantly lower than preincision values just after clamping of carotid artery in both hemispheres ( $P=0.005$ for the right and $P<0.001$ for the left side).

Conclusion: NIRS values measured in our study show that there is a correlation between hemodynamic changes and cerebral oxygenation. This effect is especially pronounced while the patient is asleep and intubated, which implies the importance of close monitoring of patients with carotid disease during any surgery requiring general anaesthesia.

Keywords: Atherosclerosis. Carotid Endarterectomy. Near-Infrared Spectroscopy. Carotid Artery, Common. Anesthesia, General.

\begin{tabular}{ll}
\hline \multicolumn{2}{l}{ Abbreviations, acronyms \& symbols } \\
\hline CC & $=$ Cross-clamping \\
CEA & $=$ Carotid endarterectomy \\
ECG & $=$ Electrocardiography \\
EEG & $=$ Electroencephalography \\
MEP & $=$ Motor-evoked potential \\
NIRS & $=$ Near-infrared spectroscopy \\
rSO & $\quad=$ Regional cerebral oxygenation \\
SPSS & $=$ Statistical Package for the Social Sciences \\
SSEP & $=$ Somatosensory-evoked potentials \\
\hline
\end{tabular}

'Department of Cardiovascular Surgery, Okmeydanı Training and Research Hospital, Istanbul, Turkey.

2Department of Anesthesia and Intensive Care, Okmeydanı Training and Research Hospital, Istanbul, Turkey.

This study was carried out at the Okmeydanı Training and Research Hospital, Istanbul, Turkey.

\section{INTRODUCTION}

Carotid endarterectomy (CEA) is a well-established procedure to prevent development of future stroke in symptomatic and asymptomatic patients with a high-grade internal carotid artery stenosis ${ }^{[1,2]}$. On the other hand, the procedure itself carries a risk of stroke, which can be due to thrombosis, embolism, or intraoperative ischemia related to hypoperfusion during crossclamping (CC) of the carotid artery ${ }^{[3]}$. Therefore, monitoring techniques to determine adequacy of cerebral circulation are used and include electroencephalography (EEG), somatosensoryevoked potentials (SSEP), motor-evoked potential (MEP), transcranial Doppler, stump pressure and cerebral oximetry ${ }^{[4]}$.
Correspondence Address:

Cihan Yücel

iD https://orcid.org/0000-0002-1941-0873

Department of Cardiovascular Surgery, Okmeydanı Training and Research Hospital. Okmeydanı Eğitim ve Araştırma Hastanesi, Kalp ve Damar Cerrahisi Poliklinigi, Kaptanpaşa Mahallesi, Darülaceze Caddesi № 25, Okmeydanı/Şişli, Istanbul, Turkey. Zip code: 34384

E-mail: cihanyucell@hotmail.com 
Near-infrared spectroscopy (NIRS) is a non-invasive technique to detect cerebral ischemia by monitoring changes in regional cerebral oxygenation $\left(\mathrm{rSO}_{2}\right)$ in the frontal lobes. NIRS is easy to apply and has a relatively low cost compared to other noninvasive techniques. Various studies compared NIRS with stump pressure ${ }^{[5,6]}, \mathrm{EEG}^{[7]}$, and transcranial Doppler ${ }^{[8]}$ and proposed it as a reliable and safe substitute for these traditional techniques. However, there are no studies showing the changes of NIRS values in response to hemodynamic variations during stages of CEA procedure and clinical implications of these changes. Some surgeons believe that an increase in mean arterial blood pressure, usually by $20 \%$ or systolic arterial blood pressure above $150 \mathrm{mmHg}$ during CC of carotid artery is quite enough to prevent cerebral ischemia. Nevertheless, there is no scientific data supporting this practice. In the current study, we evaluated the NIRS values at certain stages during carotid endarterectomy and their relation to hemodynamic changes at these time points. The aim was to determine if hemodynamic changes affect NIRS values while the other factors, such as arterial oxygen saturation, were kept relatively constant. We also intended to provide a scientific basis for the increased blood pressure protocol during carotid endarterectomy, hoping that our results will be helpful for future hemodynamic management protocols for patients with carotid stenosis.

\section{METHODS}

Between October 2018 and September 2019, 50 consecutive patients undergoing CEA under general anaesthesia were prospectively included in the study. The hospital ethics committee approved the study (EC number: 2018-973), and the individual informed consent was waived. Indications for CEA were symptomatic or asymptomatic carotid stenosis $>70 \%$ or symptomatic patients with $50-69 \%$ carotid stenosis, as stated in guidelines ${ }^{[1,2]}$ and discussed by the team. Carotid stenting was preferred over endarterectomy when revascularization is indicated in patients with cervical anatomy unfavorable to arterial surgery and at high surgical risk, but these patients were not included in the study. Patients who underwent elective carotid surgery were included and those with recent cerebral events were excluded from the sudy. Patients with either unilateral or bilateral lesions were included in the study but those with chronic total occlusion of the carotid artery were not operated on. In patients with bilateral lesions, the side of CEA was determined by the dominant hemisphere or the severity of the lesion as well as the side of symptoms, if any. The severity of the carotid artery stenosis was initially assessed by carotid Doppler ultrasound imaging and confirmed by computed tomography angiography in all patients. We included patients who underwent CEA with concomitant coronary surgery because our instituitional approach is to perform the carotid part of the operation before the coronary grafting and, therefore, measurements are logically not affected by parameters related to coronary surgery.

\section{Operative and Postoperative Protocol}

All operations were done by the same team. CEA was performed under general anesthesia in all patients in accordance to the surgeon's preference. Patients at high risk for general anaesthesia and others who underwent operation with regional anaesthesia were not included in the study to provide a more homogeneous group. Routine monitoring with ECG and pulse oximetry were used. Intra-arterial blood pressure was measured and recorded continuously using a 20-gauge catheter placed in the radial artery. Heparin (5000 U) was administered intravenously 3 minutes before CC of the carotid artery. Activated clotting time was used to monitor heparin treatment with the aim of a value above 200 seconds and an additional dose was administered when necessary. A standard longitudinal endarterectomy was performed in all patients with primary or patch closure of arteriotomy, depending on the arterial diameter and the surgeon's preference. Our strategy to use shunting is when NIRS values drop $20-25 \%$ from baseline or when the clamp time is anticipated to be long due to the anatomy of the patient and the carotid lesion. Clamp time is the duration from the start of internal carotid artery clamping to the removal of this clamp and operation time is the duration from the start of carotid surgery skin incision to the closure of this incision. In patients with concomitant coronary artery bypass grafting, CEA procedure was completed before cannulation for cardiopulmonary bypass.

Initially, patients' blood pressure was regulated and kept within normal limits and hypertension was avoided. After CC of the carotid artery, NIRS at first minute was measured and then systolic blood pressure was increased above $150 \mathrm{mmHg}$ and vasoactive medications were used, if required. Ephedrine was used as the preferred vasoactive medication ${ }^{[9]}$. After CC was removed, blood pressure was regulated to keep it within normal limits. Patients were extubated after the return of spontaneous respiration and were transferred to the intensive care unit for follow-up on the first night. All patients were on dual antiplatelet therapy, with $75 \mathrm{mg}$ of acetylsalicylic acid and clopidogrel, in the postoperative period.

\section{NIRS Measurements}

Continuous bilateral $\mathrm{rSO}_{2}$ measurements were performed using a Masimo Cerebral Oximeter (Masimo Corp, CA, USA), by 2 sensors placed on the forehead. NIRS values from both hemispheres were recorded simultaneously at certain time points and were compared with each other to evaluate the changes at different stages of operation as follows: before the start of the operation, when the patient was awake, after the patient is asleep just before incision, at the first minute of CC of carotid artery, just after increasing blood pressure, every 5 minutes after CC, after removal of CC and when the patient is awake and extubated. All these values were compared ipsilaterally with each other (right NIRS vs. right NIRS) and also right and left NIRS values were compared with each other at each time point.

\section{Statistical Analysis}

Statistical analysis was performed with IBM SPSS software version 20 (IBM, Armonk, NY, USA). All data are presented as mean \pm standard deviation for continuous variables, as numbers with percentages for categorical variables. The Shapiro-Wilk test was used to evaluate the normality of variable distribution. 
In the comparison of two independent groups, the Student's t-test was used for numerical variables with normal distribution. The correlations of systolic, diastolic and mean pressures with NIRS at different time points were investigated using Pearson's correlation coefficient and Bland-Altman analysis. A $P$ value of 0.05 or less was considered significant.

\section{RESULTS}

\section{Patient Characteristics}

A total of 50 consecutive patients who underwent CEA under general anaesthesia between October 2018 and September 2019 were prospectively included in the study. Data for all patients were collected prospectively and were complete. The mean age was 69.04 \pm 6.6 (range, 54-82 years) and most patients had a risk factor for atherosclerosis (Table 1). Most patients had CEA for symptomatic carotid disease (stroke, 38\% or transient ischemic attack, 20\%), as shown in Table 1. Concomitant coronary artery bypass grafting was performed in 6 (12\%) patients.

\section{Perioperative Data}

All patients underwent either right (44\%) or left (56\%) CEA under general anaesthesia. In 18 patients with bilateral lesions, a left CEA was performed in most patients (14). Primary closure of the arteriotomy was preferred in most patients (78\%) and shunt was not used in any patient. Postoperatively, there was transient hemiparesis in 1 patient and local hematoma of the surgical site was observed in 4 patients, 2 of whom required re-exploration.
The patient with transient hemiparesis underwent left CEA and woke from anaesthesia with right-sided hemiparesis that lasted 2 days. At the time of operation of this patient, NIRS values showed a decline of about 25\% from baseline 5 minutes after clamping, but it was not possible to insert a shunt due to anatomical problems. The CT scan of this patient showed no pathology and the patient was discharged without any neurological deficits. There were no major stroke, nerve injury or death in any other patient. Surgical and early postoperative data are summarized in Table 2.

\section{NIRS Assessments}

NIRS values of the right and left hemispheres were compared with each other and were not different from each other at any of the time points (Table 3). We also analyzed these differences in patients with either right, left or bilateral lesions and could not find any difference at any time point, so we did not present them in the table to simplify the interpretation of results.

NIRS values were observed to drop significantly from preincision values just after CC of carotid artery in both hemispheres ( $P=0.005$ for the right side and $P<0.001$ for the left side). At this point, blood pressures were being regulated to keep them within normotensive limits. Systolic blood pressures increased above $150 \mathrm{mmHg}$ and NIRS values were observed to increase immediately and were comparable to values before intubation and incision, as seen in Table 3. At the $5^{\text {th }}$ minute after CC, NIRS values were observed to fall from the values in systolic blood pressures of $150 \mathrm{mmHg}$ ( $P=0.8$ for the right side and $P<0.001$ for

Table 1. Preoperative patient data.

\begin{tabular}{|c|c|c|c|}
\hline Age (years) & & $69.04 \pm 6.6$ & $54-82$ \\
\hline Ejection fraction & & $55.4 \pm 5.7$ & $45-65$ \\
\hline Variable & & $\mathbf{n}$ & $\%$ of total \\
\hline \multirow{2}{*}{ Sex } & Male & 34 & 32 \\
\hline & Female & 16 & \\
\hline \multicolumn{2}{|l|}{ Hypertension } & 37 & 74 \\
\hline \multicolumn{2}{|l|}{ Smoking } & 33 & 66 \\
\hline \multicolumn{2}{|l|}{ Diabetes mellitus } & 23 & 46 \\
\hline \multicolumn{2}{|l|}{ Coronary artery disease } & 10 & 20 \\
\hline \multicolumn{2}{|l|}{ Concomitant CABG } & 6 & 12 \\
\hline \multicolumn{2}{|l|}{ COPD } & 11 & 22 \\
\hline \multirow{3}{*}{ Symptom } & Stroke & 19 & 38 \\
\hline & TIA & 10 & 20 \\
\hline & Asymptomatic & 21 & 42 \\
\hline \multicolumn{2}{|l|}{ Bilateral lesion } & 18 & 36 \\
\hline
\end{tabular}

$\mathrm{CABG}=$ coronary artery bypass grafting; $\mathrm{COPD}=$ chronic obstructive pulmonary disease; $\mathrm{TI} A=$ transient ischemic attack 
Table 2. Operative and postoperative patient data.

\begin{tabular}{|c|c|c|c|}
\hline & Variable & $\mathbf{n}$ & $\%$ of total \\
\hline \multirow{2}{*}{ Side of endarterectomy } & Right & 22 & 44 \\
\hline & Left & 28 & 56 \\
\hline \multirow{2}{*}{ Arteriotomy closure } & Primary & 39 & 78 \\
\hline & Patch & 11 & 22 \\
\hline \multicolumn{2}{|l|}{ Shunt use } & 0 & 0 \\
\hline \multirow{5}{*}{ Postoperative complications } & Permanent cerebrovascular event & 0 & 0 \\
\hline & Transient hemiparesis & 1 & 2 \\
\hline & Local hematoma & 4 & 8 \\
\hline & Exploration for bleeding/hematoma & 2 & 4 \\
\hline & Mortality & 0 & 0 \\
\hline \multicolumn{2}{|l|}{ Variable } & \multicolumn{2}{|c|}{ Mean $\pm S D$ (range) } \\
\hline \multicolumn{2}{|l|}{ Clamp duration (minutes) } & \multicolumn{2}{|c|}{$21.7 \pm 6.3(9-33)$} \\
\hline \multicolumn{2}{|l|}{ ICU stay (days) } & \multicolumn{2}{|c|}{$1.1 \pm 0.5(1-3)$} \\
\hline \multicolumn{2}{|l|}{ Hospital stay (days) } & \multicolumn{2}{|c|}{$3.3 \pm 1.3(2-8)$} \\
\hline
\end{tabular}

ICU=intensive care unit

the left side). However, they increased and were stable after 10 minutes of CC of carotid artery. In Table 3, 15-minute values were given as representative of these similar and stable values.

Although NIRS values decreased significantly after CC, in only six patients they dropped to more than $20 \%$ of baseline values. Three of these patients underwent right CEA, 3 underwent left CEA and only 1 had bilateral lesions. The decrease in NIRS values was observed ipsilateral to CEA in all. In 5 of these patients, after the increase in systolic blood pressure levels, NIRS values increase to levels above $80 \%$ of the initial measurements. In 1 patient, NIRS level remained low, but due to technical reasons, it was not possible to insert a shunt and this was our only patient with postoperative transient hemiparesis.

After completing the procedure and removing CC of carotid arteries, NIRS values increased to pre-incision levels. When compared to preoperative awake values, postoperative awake values did not increase significantly on the right side, but were found to be increased on the left $(P=0.02)$.

Correlation of NIRS values with blood pressure indices at different time points was evaluated to assess if hemodynamic changes are more effective at any stage of the operation. Rightand left-sided NIRS values were well correlated with systolic, diastolic and mean blood pressures when the patient is asleep and intubated (Figures 1 and 2). When patients were awake or when carotid artery was clamped during surgery, the correlations were not statistically significant.

\section{DISCUSSION}

Cerebral oximetry measures the ratio of oxygenated hemoglobin to total hemoglobin and the results can be influenced by many factors, including head position, mean arterial pressure, systemic arterial blood oxygen saturation and partial pressure of carbon dioxide, hemoglobin concentration, brain tissue oxygen consumption, and intracranial pressure ${ }^{[10]}$. Nevertheless, it has been used as a reliable and safe substitute for traditional brain monitoring techniques. Wang et al. ${ }^{[1]}$ found that the sensitivity and specificity of NIRS monitoring for intraoperative hypoperfusion were $64.3 \%$ and $90.0 \%$, resulting in a strong consistency with transcranial Doppler ultrasonography monitoring results. Jonsson et al. ${ }^{[5]}$ compared stump pressures and NIRS measurements in CEA operations in terms of shunt placement and reported that NIRS monitoring has a high sensitivity and acceptable specificity in predicting cerebral ischaemia and can be an attractive alternative for stump pressure measurement. One of the major advantages of NIRS monitoring is the spontaneous observation of the changes in cerebral oxygenation in accordance to changes in cerebral circulation. These properties, along with its ease of use, rendered NIRS monitoring a very suitable tool for this study, because it was possible to assess spontaneous changes in cerebral oxygenation in response to hemodynamic changes.

In our institution, carotid operations are performed under either general or local anaesthesia with sedation. There are 
Table 3. Comparison of NIRS values at different time points.

\begin{tabular}{|c|c|c|c|}
\hline Time NIRS measured & Right-sided NIRS & Left-sided NIRS & $P$-value \\
\hline Before intubation & $60.5 \pm 5.3$ & $59.7 \pm 3.7$ & 0.2 \\
\hline Before incision & $59.3 \pm 7.5$ & $60.4 \pm 7.5$ & 0.1 \\
\hline$P$-value & 0.2 & 0.4 & \\
\hline Before incision & $59.3 \pm 7.5$ & $60.4 \pm 7.5$ & 0.1 \\
\hline 1 minute after clamping & $56.7 \pm 8.3$ & $56.5 \pm 8.4$ & 0.8 \\
\hline P-value & 0.005 & $<0.001$ & \\
\hline 1 minute after clamping & $56.7 \pm 8.3$ & $56.5 \pm 8.4$ & 0.8 \\
\hline After SBP above 150 mmHg & $58.5 \pm 7.9$ & $57.7 \pm 10.1$ & 0.4 \\
\hline P-value & 0.001 & 0.2 & \\
\hline Before intubation & $60.5 \pm 5.3$ & $59.7 \pm 3.7$ & 0.2 \\
\hline After SBP above 150 mmHg & $58.5 \pm 7.9$ & $57.7 \pm 10.1$ & 0.4 \\
\hline$P$-value & 0.054 & 0.1 & \\
\hline After SBP above 150 mmHg & $58.5 \pm 7.9$ & $57.7 \pm 10.1$ & 0.4 \\
\hline 5 minutes after clamping & $56.6 \pm 7.8$ & $55.4 \pm 10.1$ & 0.2 \\
\hline$P$-value & 0.8 & $<0.001$ & \\
\hline 5 minutes after clamping & $56.6 \pm 7.8$ & $55.4 \pm 10.1$ & 0.2 \\
\hline 15 minutes after clamping & $58.3 \pm 7.6$ & $59.1 \pm 8.8$ & 0.4 \\
\hline$P$-value & $<0.001$ & $<0.001$ & \\
\hline Before incision & $59.3 \pm 7.5$ & $60.4 \pm 7.5$ & 0.1 \\
\hline After removing clamp & $59.8 \pm 7.4$ & $60.2 \pm 8.3$ & 0.5 \\
\hline P-value & 0.5 & 0.7 & \\
\hline Before intubation & $60.5 \pm 5.3$ & $59.7 \pm 3.7$ & 0.2 \\
\hline After extubation & $62.3 \pm 7.6$ & $62.6 \pm 7.2$ & 0.6 \\
\hline$P$-value & 0.07 & 0.002 & \\
\hline
\end{tabular}

Before incision=patient is asleep and intubated; Before intubation=patient is awake; NIRS=near-infrared spectroscopy; SBP=systolic blood pressure

many factors affecting NIRS values as mentioned above and most of these, such as oxygen saturation and partial pressure of carbon dioxide in systemic arterial blood, as well as blood pressure can be easily controlled under general anaesthesia. Therefore, patients undergoing CEA under general anaesthesia created a homogenous group to analyze mostly the effects of hemodynamic changes on NIRS and were specifically selected for this study. Further studies including patients undergoing surgery with local anaesthesia may be performed and may give further insight about the cerebral oxygenation during various stages of carotid surgery in different patient groups.
In this study, NIRS values were only found to be correlated with blood pressure indices when the patient was under general anaesthesia (Figures 1 and 2). This is an important finding that shows that regulatory mechanisms of cerebral blood flow are less effective and cerebral oxygenation is more dependent on hemodynamic changes in patients under general anaesthesia. It is not possible to compare this result with any other, since there are no studies in the literature that evaluate the effects of hemodynamic parameters on cerebral oxygenation by any methods of cerebral monitoring. This study is unique in this regard and may lead to future studies that use and compare 


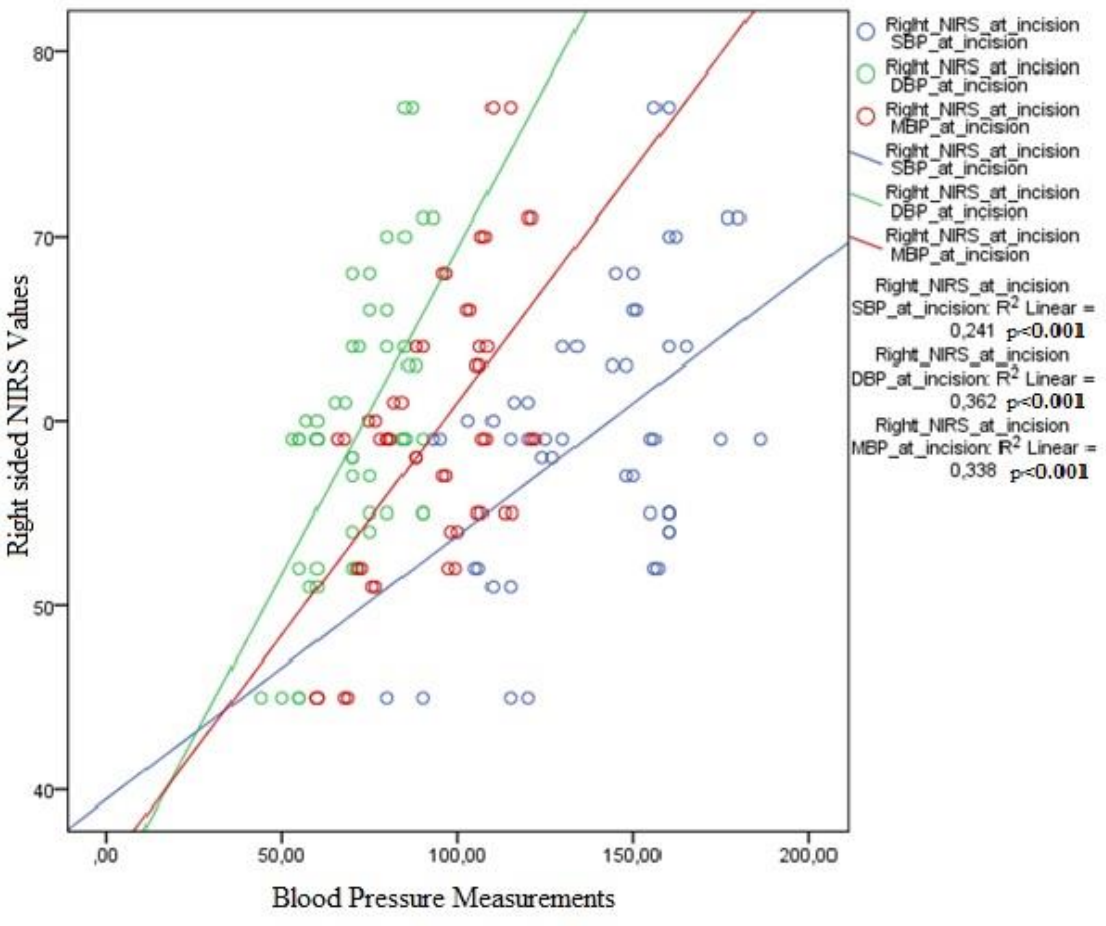

Fig. 1 - Correlation of right-sided NIRS values with blood pressure indices after general anaesthesia.

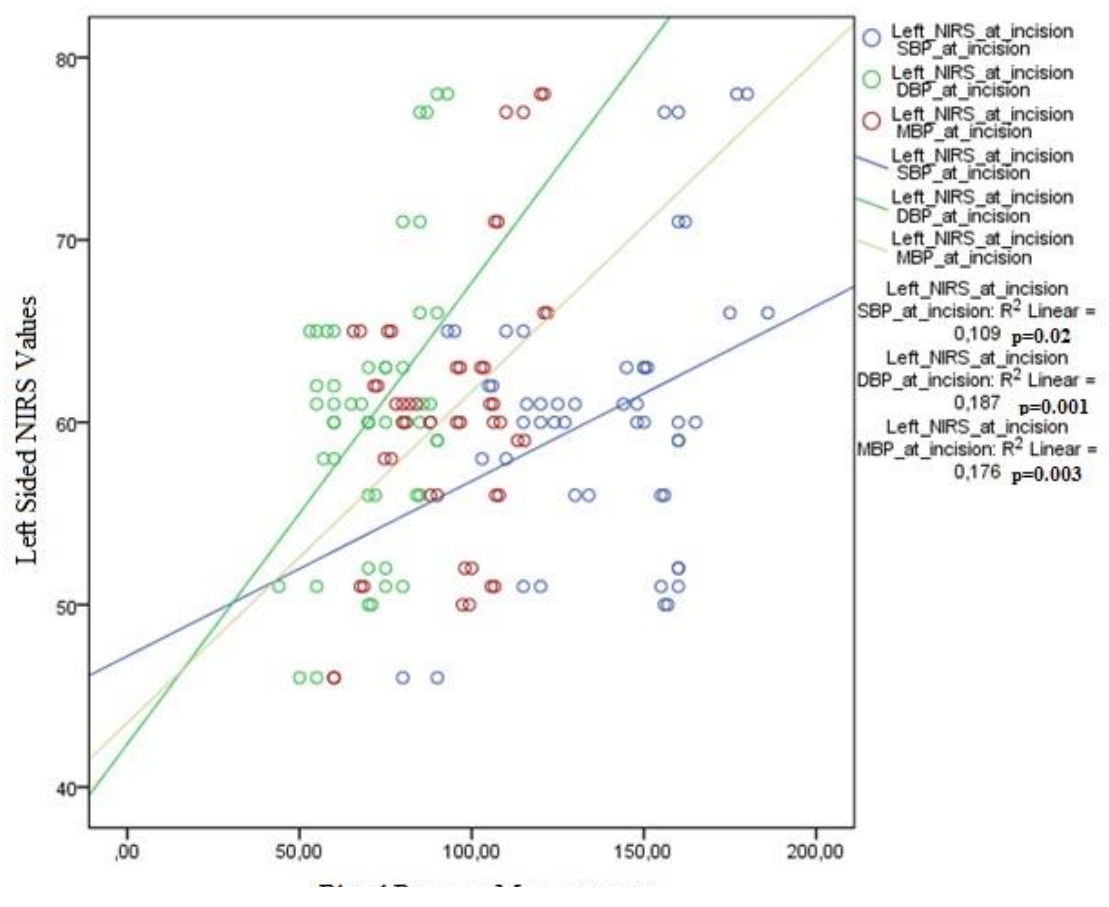

Fig. 2 - Correlation of left-sided NIRS values with blood pressure indices after general anaesthesia. different methods of cerebral monitoring to assess factors affecting cerebral oxygenation in patients with carotid disease undergoing any surgery under general anaesthesia.

A time-honored approach to maintain cerebral perfusion during CC of the carotid artery is to increase mean arterial blood pressure, usually by $20 \%$ or systolic blood pressure above $150 \mathrm{mmHg}$. Although this is a general discussion point among surgeons, there are no studies in the literature that quantitatively assess the effect of increased blood pressure on cerebral perfusion during carotid artery clamping. This study showed that initial decrease in NIRS values after CC was restored by increasing systolic blood pressure above $150 \mathrm{mmHg}$ (Table 3). This suggests that blood pressure has an influence on brain oxygenation whereas NIRS values did not correlate with blood pressure indices after CC of the carotid artery in our analysis. This may be because systolic blood pressures were kept stable and around $150-160 \mathrm{mmHg}$ in all our patients from this time until the removal of carotid clamp. Since there are no comparable studies, this may be evaluated in another study without strict control of blood pressure after CC of the carotid artery.

There was a second decrease in the NIRS values on the left side 5 minutes after clamping, which increased in a very short period and remained so till the clamp was removed. These values are all analyzed on a minute basis and the representative values are presented in Table 3. They remain stable afterwards at stable blood pressures, which suggests that local regulation of cerebral blood flow may also have a role. The reason for observing this only on the left side is difficult to interpret, but it may be the result of more patients undergoing CEA on the left carotid artery.

Although this study showed a positive relationship between hemodynamic parameters and NIRS values, the clinical implications of these findings are still controversary. Percent changes in NIRS values after CC of carotid artery and increased blood pressures were relatively small in our study. The cut-off value of NIRS varies among different clinical settings. Although a 12\% decrease in $\mathrm{rSO}_{2}$ was reported to have a higher sensitivity, specificity, and predictive values than a $20 \%$ decrease in $\mathrm{rSO}_{2}{ }^{[12]}$, it was recently agreed that more than a $20 \%$ decrease in $\mathrm{rSO}_{2}$ compared to the baseline 
value indicates a need for intervention ${ }^{[13,14]}$ and could be considered as a cut-off value to indicate cerebral ischemia $\left.{ }^{[15}\right]$. We observed a decrease in NIRS values of more than $20 \%$ in 6 patients and in 5 of them this percentage decreased to less than 20\% after increasing blood pressure and in the remaining patient we observed a neurological complication. Although our numbers are low to achieve a statistical significance, we can suggest that decreased NIRS values may indicate a neurological complication if they remain below $20 \%$ of baseline values after the increase in blood pressure.

Another finding in the present study was the correlation of the right- and left-sided NIRS values with each other, regardless of the side of the lesion. This was true for all time points of measurement, including those after clamping. Moreover, 18 patients with bilateral lesions showed similar pattern of NIRS changes to the patients with unilateral lesions (e.g. right- and left-sided NIRS in those with bilateral lesions; at incision, 60 \pm 7.5

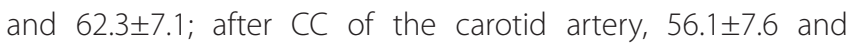
$56.2 \pm 7.6)$. Only in those patients with more than $20 \%$ decrease in NIRS values, the decrease was ipsilateral to the clamped carotid artery in all. It is possible that this decrease occurs in patients with problems of intracerebral circulation and in patients with an intact circle of Willis, the cerebral oxygenation is affected bilaterally or globally after clamping one side, regardless of the side. An anatomical study with a larger number of patients that includes screening the completeness of circle of Willis is necessary to prove this finding.

One important shortcoming of this study is the relatively small number of patients. Since the number of postoperative neurological complications is very low, it is also not possible to interpret the full clinical implication of our findings. Moreover, there are no similar studies to compare our results, which on the other hand makes the study unique. Authors are also aware that NIRS monitoring has its shortcomings and the results may be affected by many factors, as stated. However, this is a preliminary study with important results and we believe it will lead to further studies to assess the factors affecting cerebral oxygenation during carotid endarterectomy and their clinical implications.

\section{CONCLUSION}

As an acceptable monitoring tool during CEA, NIRS values show a correlation between hemodynamic changes and cerebral oxygenation. This effect is especially pronounced while the patient is asleep and intubated, which implicates the importance of close monitoring of patients with cartotid disease during any surgery that requires general anaesthesia.

\section{No financial support.}

No conflict of interest.

\section{Authors' roles \& responsibilities}

$\mathrm{CY}$ Substantial contributions to the conception or design of the
work; or the acquisition, analysis, or interpretation of data for
the work; drafting the work or revising it critically for important
intellectual content; agreement to be accountable for all aspects
of the work in ensuring that questions related to the accuracy or
integrity of any part of the work are appropriately investigated
and resolved; final approval of the version to be published

SK

Substantial contributions to the conception or design of the work; or the acquisition, analysis, or interpretation of data for the work; drafting the work or revising it critically for important intellectual content; agreement to be accountable for all aspects of the work in ensuring that questions related to the accuracy or integrity of any part of the work are appropriately investigated and resolved; final approval of the version to be published

MG

Substantial contributions to the conception or design of the work; or the acquisition, analysis, or interpretation of data for the work; drafting the work or revising it critically for important intellectual content; agreement to be accountable for all aspects of the work in ensuring that questions related to the accuracy or integrity of any part of the work are appropriately investigated and resolved; final approval of the version to be published

ST

Substantial contributions to the conception or design of the work; or the acquisition, analysis, or interpretation of data for the work; drafting the work or revising it critically for important intellectual content; agreement to be accountable for all aspects of the work in ensuring that questions related to the accuracy or integrity of any part of the work are appropriately investigated and resolved; final approval of the version to be published

NK

Substantial contributions to the conception or design of the work; or the acquisition, analysis, or interpretation of data for the work; drafting the work or revising it critically for important intellectual content; agreement to be accountable for all aspects of the work in ensuring that questions related to the accuracy or integrity of any part of the work are appropriately investigated and resolved; final approval of the version to be published

\section{REFERENCES}

1. Naylor AR, Ricco JB, de Borst GJ, Debus S, de Haro J, Halliday A, et al. Editor's choice - Management of atherosclerotic carotid and vertebral artery disease: 2017 clinical practice guidelines of the European society for vascular surgery (ESVS). Eur J Vasc Endovasc Surg. 2018;55(1):3-81. doi:10.1016/j.ejvs.2017.06.021.

2. Aboyans V, Ricco JB, Bartelink MEL, Björck M, Brodmann M, Cohnert T,

et al. 2017 ESC guidelines on the diagnosis and treatment of peripheral arterial diseases, in collaboration with the European society for vascular surgery (ESVS): document covering atherosclerotic disease of extracranial carotid and vertebral, mesenteric, renal, upper and lower extremity arteries endorsed by: the European stroke organization (ESO) the task force for the diagnosis and treatment of peripheral arterial 
diseases of the European society of cardiology (ESC) and of the European society for vascular surgery (ESVS). Eur Heart J. 2018;39(9):763-816. doi:10.1093/eurheartj/ehx095.

3. de Borst GJ, Moll FL, van de Pavoordt HD, Mauser HW, Kelder JC, Ackerstaf RG. Stroke from carotid endarterectomy: when and how to reduce perioperative stroke rate? Eur J Vasc Endovasc Surg. 2001;21 (6):484-9. doi:10.1053/ejvs.2001.1360.

4. Lam AM, Kianpour D. Monitoring for carotid endarterectomy: more or less? Anesth Analg. 2015;120(6):1186-8. doi:10.1213/ ANE.0000000000000734.

5. Jonsson M, Lindström D, Wanhainen A, Djavani Gidlund K, Gillgren P. Near infrared spectroscopy as a predictor for shunt requirement during carotid endarterectomy. Eur J Vasc Endovasc Surg. 2017;53(6):783-91. doi:10.1016/j.ejvs.2017.02.033.

6. Kragsterman B, Pärsson H, Bergqvist D. Local haemodynamic changes during carotid endarterectomy--the influence on cerebral oxygenation. Eur JVasc Endovasc Surg. 2004;27(4):398-402. doi:10.1016/j. ejvs.2004.01.024.

7. Pennekamp CW, Immink RV, den Ruijter HM, Kappelle LJ, Bots ML, Buhre WF, et al. Near-infrared spectroscopy to indicate selective shunt use during carotid endarterectomy. Eur J Vasc Endovasc Surg. 2013;46(4):397-403. doi:10.1016/j.ejvs.2013.07.007.

8. Cho JW, Jang JS. Near-infrared spectroscopy versus transcranial Dopplerbased monitoring in carotid endarterectomy. Korean JThorac Cardiovasc Surg. 2017;50(6):448-52. doi:10.5090/kjtcs.2017.50.6.448.

9. Aliane J, Dualé C, Guesmi N, Baud C, Rosset E, Pereira B, et al. Compared effects on cerebral oxygenation of ephedrine vs phenylephrine to treat hypotension during carotid endarterectomy. Clin Exp Pharmacol Physiol. 2017;44(7):739-48. doi:10.1111/1440-1681.12759.

10. Denault A, Deschamps A, Murkin JM. A proposed algorithm for the intraoperative use of cerebral near-infrared spectroscopy. Semin Cardiothorac Vasc Anesth. 2007;11(4):274-81. doi:10.1177/1089253207311685.

11. Wang Y, Li L, Wang T, Zhao L, Feng H, Wang Q, et al. The efficacy of near-infrared spectroscopy monitoring in carotid endarterectomy: a prospective, single-center, observational study. Cell Transplant. 2019;28(2):170-5. doi:10.1177/0963689718817760.

12. Mille T, Tachimiri ME, Klersy C, Ticozzelli G, Bellinzona G, Blangetti I, et al. Near infrared spectroscopy monitoring during carotid endarterectomy: which threshold value is critical? Eur JVasc Endovasc Surg. 2004;27(6):646-50. doi:10.1016/j.ejvs.2004.02.012.

13. Mohandas BS, Jagadeesh AM, Vikram SB. Impact of monitoring cerebral oxygen saturation on the outcome of patients undergoing open heart surgery. Ann Card Anaesth. 2013;16(2):102-6. doi:10.4103/09719784.109740.

14. Colak Z, Borojevic M, Bogovic A, Ivancan V, Biocina B, MajericKogler $V$. Influence of intraoperative cerebral oximetry monitoring on neurocognitive function after coronary artery bypass surgery: a randomized, prospective study. Eur J Cardiothorac Surg. 2015;47(3):44754. doi:10.1093/ejcts/ezu193.

15. Zogogiannis ID, latrou CA, Lazarides MK, Vogiatzaki TD, Wachtel MS, Chatzigakis PK, et al. Evaluation of an intraoperative algorithm based on near-infrared refracted spectroscopy monitoring, in the intraoperative decision for shunt placement, in patients undergoing carotid endarterectomy. Middle East J Anaesthesiol. 2011;21(3):367-73. 